\title{
Studies on Frequency Distribution, Skewness and Kurtosis in F1m1 Mutant Populations of Sesame
}

\author{
Rajesh Kumar Kar*, Tapash Kumar Mishra and Banshidhar Pradhan \\ Department of Plant Breeding and Genetics, College of Agriculture, \\ OUAT, Bhubaneswar-751003, India \\ *Corresponding author
}

\section{Keywords}

Ethyl methane sulfonate, F1M1 mutants, Frequency distribution, Kurtosis, Sesame and Skewness

Article Info

Accepted:

15 March 2019

Available Online:

10 April 2019

\section{A B S T R A C T}

The present study in sesame was undertaken to make an assessment of the combined effects of hybridization and ethyl methanesulfonate (EMS) on parameters like frequency distribution, skewness and kurtosis of quantitative traits related to productivity in $\mathrm{F}_{1} \mathrm{M}_{1}$ generation. Total nine populations (three EMS treated population $\left(\mathrm{F}_{1}{ }^{1} \mathrm{M}_{1}\right)$, their respective controls $\left(\mathrm{F}_{1 \mathrm{~s}}\right)$ and three varieties) were grown in a randomized block design (RBD) with three replications during summer, 2017. Observations were taken on traits, viz., plant height, number of primary branches per plant, number of capsules per plant and single plant yield. Population ${ }^{1} \mathrm{~F}_{1}{ }^{1} \mathrm{M}_{1}$ (mutant population of Nirmala $\mathrm{X}$ Prachi) and ${ }^{3} \mathrm{~F}_{1}{ }^{1} \mathrm{M}_{1}$ (mutant population of Prachi $\mathrm{X}$ Amrit) showed slightly negative spread and flat distribution. Frequency curves showed more or less restricted distribution in mutant population for primary branches per plant than their respective controls. In frequency curves mutant population showed slight greater spread than their respective controls for number of capsules per plant. All the three $\mathrm{F}_{1}{ }^{1} \mathrm{M}_{1 \mathrm{~s}}$ gave a slight positive skewness for number of capsules per plant. Population ${ }^{1} \mathrm{~F}_{1}{ }^{1} \mathrm{M}_{1}$ was found to be platykurtic. The distribution of seed yield per plant appeared to be positive skewness, longer tail to the right in all the $\mathrm{F}_{1}{ }^{1} \mathrm{M}_{1 \mathrm{~s}}$ this is also apparent from the frequency curves. The changes in mutant populations compared to the respective controls for different characters must have come through induced micro mutations. However, these changes would be better known from analyses in later generations.

\section{Introduction}

Sesame (Sesamum indicum), a diploid species with $2 \mathrm{n}=26$ chromosomes is an ancient oil yielding crop. In 2016, world production of sesame seeds was 6.1 million tones and leading sesame producing countries were Tanzania, Myanmar, India, and Sudan
(FAOSTAT, 2017). Sesame is cultivated in an area of 19.53 lakh hectares in India with an annual production of 8.32 lakh tonnes and productivity of $426.4 \mathrm{~kg} / \mathrm{ha}$ (Anonymous, 2015-16). Uttar Pradesh, Gujarat, Madhya Pradesh, Tamil Nadu, Odisha, Andhra Pradesh, West Bengal and Rajasthan are the major sesame growing states. Productivity of 
the crop varies considerably from state to state. The low productivity of sesame in India is due to low yield potential of the varieties coupled with poor crop management. Genetic variation is a pre-requisite for selection to be effective. Achievement in any breeding programmes depends on the amount as well as the nature of variation. Hybridization followed by selection in segregating generations is the main approach in breeding in self-pollinated crops. But tight linkage of polygenes controlling the characters of significance could be a barrier to release the useful variability. To overcome this barrier some workers have examined the usefulness of combining hybridization and mutagenesis for generating variability for productivity traits. Chemical mutagen treatment to the hybrids is one of the methods of realizing increased mutation frequency. Studies on the efficacy of chemical mutagen treatment (EMS) in hybrid material for generating useful variability in sesame are extremely limited. The present investigation intended at analyzing the change in distribution pattern in the $\mathrm{F}_{1} \mathrm{M}_{1}$ mutant populations due to induction of micromutations in traits and to assess the scope of improvement.

\section{Materials and Methods}

The fundamental material of the present study consisted of three adapted sesame varieties of Odisha viz., Nirmala, Prachi and Amrit. These varieties were crossed and three intervarietal crosses $\left(\mathrm{F}_{1 \mathrm{~s}}\right)$ were produced excluding the reciprocal crosses. Based on the previous reported research, a dose rate of $0.5 \%$ ethyl methanesulfonate (for three hours) was selected for treatment of material of the present study (Begum and Dasgupta, 2010, Begum and Dasgupta, 2015 and Kumari et al., 2016). For ethyl methanesulfonate (EMS) treatment, the three $F_{1 s}$ seeds were separately presoaked in distilled water overnight and then treated with 0.5 per cent freshly preparer solutions for 3 hours. The treated seeds were washed thoroughly for $1 \mathrm{~h}$ in running tap water to remove the residual effect of the mutagenic chemicals. The treated seeds were sown instantly in the field along with varieties and their respective controls to grow the $\mathrm{F}_{1} \mathrm{M}_{1}$ generation in a randomized block design (RBD) with three replications during summer, 2017. Designation of various treatments and controls $\left(\mathrm{F}_{1 \mathrm{~s}}\right)$ in $\mathrm{F}_{1} \mathrm{M}_{1}$ generation is presented in Table 2. The experimental plot consisted of 7 rows of $3 \mathrm{~m}$ each. Sowing was done with 30 $\mathrm{cm} \times 10 \mathrm{~cm}$ spacing. Designation of various treatments and controls $\left(\mathrm{F}_{1 \mathrm{~s}}\right)$ in $\mathrm{F}_{1} \mathrm{M}_{1}$ generation is presented in Table 1. In order to ensure stand, 2-3 seeds were dibbled per hill and later thinned one seedling per hill. Normal cultural practices including' fertilizer application@ @ $30 \mathrm{~kg} \mathrm{~N}, 60 \mathrm{~kg} \mathrm{P}_{2} \mathrm{O}_{5}$ and $40 \mathrm{~kg}$ $\mathrm{K}_{2} \mathrm{O}$ and plant protection measures were followed. Observations were taken on four quantitative traits, viz., plant height, number of primary branches per plant, number of capsules per plant and single plant yield. Single plant observation was taken for all the character using a sample of 30 randomly chosen competitive plants per plot. The material was studied for polygenic variability in $\mathrm{F}_{1}{ }^{1} \mathrm{M}_{1}$ along with corresponding controls.

The pattern of variation in the treated populations was examined through frequency curves. Statistical analysis for frequency curves was performed in Microsoft Excel 2007 (Microsoft Corp., Redmond, WA, USA). Possible deviation from normality was examined through estimates of skewness and kurtosis as suggested by Gupta, 1981.

\section{Results and Discussion}

The pattern of variation of yield and three other characters, i.e. plant height, number of primary branches per plant and number of capsules per plant in $\mathrm{F}_{1} \mathrm{M}_{1}$ populations was analysed by frequency curves. This was also 
supplemented by estimates of skewness and kurtosis, which are measures of deviation from normality. Frequency curves of the $\mathrm{F}_{1} \mathrm{M}_{1}$ populations for the above mentioned characters are presented in Figure 1-12. Each figure gives the frequency curves of the two kind of $F_{1} M_{1}$ populations following one cycle, EMS treatment of hybrid and the relevant control $\left(F_{1}\right)$ for one character. The estimates of skewness and kurtosis for all the four characters examined for pattern of variation are presented in Table 2.

In the frequency curves population ${ }^{1} \mathrm{~F}_{1}{ }^{1} \mathrm{M}_{1}$ (mutant population of Nirmala $\mathrm{X}$ Prachi) and
${ }^{3} \mathrm{~F}_{1}{ }^{1} \mathrm{M}_{1}$ (mutant population of Prachi X Amrit) showed slightly negative spread and flat distribution but population ${ }^{2} \mathrm{~F}_{1}{ }^{1} \mathrm{M}_{1}$ (mutant population of Nirmala X Amrit) appeared to be similar than their respective controls. Skewness of plant height ranged from - 0.448 to 0.289 in the mutant population. All $\mathrm{F}_{1}{ }^{1} \mathrm{M}_{1 \mathrm{~s}}$ gave slight negative skewness estimates, indicating longer tail to the left and distribution fairly symmetrical distribution for plant height. $F_{1}{ }^{1} M_{1}$ of Nirmala $X$ Prachi showed mesokurtic distribution and rest $\mathrm{F}_{1}{ }^{1} \mathrm{M}_{1 \mathrm{~s}}$ showed the slightly platykurtic distribution for plant height.

Table.1 Designation of various treatments and controls in F1M1 generation

\begin{tabular}{|l|l|}
\hline $\begin{array}{l}\text { Treatment } \\
\text { symbol }\end{array}$ & Description \\
\hline $\mathbf{V}_{\mathbf{1}}$ & Variety 1 (Nirmala) \\
\hline $\mathbf{V}_{\mathbf{2}}$ & Variety 2 ( Prachi ) \\
\hline $\mathbf{V}_{\mathbf{3}}$ & Variety 3 ( Amrit ) \\
\hline${ }^{1} \mathbf{F}_{\mathbf{1}}$ & $\mathrm{F}_{1}$ generation of Cross 1 \\
\hline${ }^{2} \mathbf{F}_{\mathbf{1}}$ & $\mathrm{F}_{1}$ generation of Cross 2 \\
\hline${ }^{3} \mathbf{F}_{\mathbf{1}}$ & $\mathrm{F}_{1}$ generation of Cross 3 \\
\hline${ }^{1} \mathbf{F}_{\mathbf{1}} \mathbf{M}_{\mathbf{1}}$ & $\mathrm{M}_{1}$ generation following chemical mutagen treatment of $\mathrm{F}_{1}$ seed of Cross 1 \\
\hline${ }^{2} \mathbf{F}_{1} \mathbf{M}_{\mathbf{1}} \mathbf{M}_{\mathbf{1}}$ & $\mathrm{M}_{1}$ generation following chemical mutagen treatment of $\mathrm{F}_{1}$ seed of Cross 2 \\
\hline${ }^{3} \mathbf{F}_{\mathbf{1}} \mathbf{M}_{\mathbf{1}}$ & $\mathrm{M}_{1}$ generation following chemical mutagen treatment of $\mathrm{F}_{1}$ seed of Cross 1 \\
\hline
\end{tabular}

The superscript of $\mathrm{F}_{1}$ is not a part of Filial generation notation

Table.2 Skewness (Sk) and Kurtosis (kts) estimates for four characters in F1M1 generation

\begin{tabular}{|l|l|l|l|l|l|l|l|l|}
\hline Treatments & \multicolumn{2}{|l|}{ Plant height } & \multicolumn{2}{|l|}{$\begin{array}{l}\text { Number of primary } \\
\text { branches per plant }\end{array}$} & $\begin{array}{l}\text { Number } \\
\text { capsules } \\
\text { plant }\end{array}$ & $\begin{array}{r}\text { of } \\
\text { per }\end{array}$ & $\begin{array}{l}\text { Seed yield per } \\
\text { plant }\end{array}$ & \\
\hline & Sk & Kts & Sk & Kts & Sk & Kts & Sk & Kts \\
\hline $\mathbf{V}_{\mathbf{1}}$ & 0.031 & 2.444 & 0.751 & 3.372 & 0.787 & 3.040 & 1.168 & 3.740 \\
\hline $\mathbf{V}_{\mathbf{2}}$ & -0.376 & 5.019 & 0.160 & 2.075 & 0.415 & 2.599 & 0.388 & 2.709 \\
\hline $\mathbf{V}_{\mathbf{3}}$ & -0.191 & 2.159 & 0.811 & 3.040 & 0.642 & 2.650 & 0.798 & 3.281 \\
\hline${ }^{\mathbf{1}} \mathbf{F}_{\mathbf{1}}$ & -0.191 & 3.681 & 0.199 & 2.363 & 0.771 & 3.459 & 1.400 & 5.271 \\
\hline${ }^{\mathbf{1}} \mathbf{F}_{\mathbf{1}} \mathbf{M}_{\mathbf{1}} \mathbf{M}_{\mathbf{1}}$ & -0.050 & 3.181 & 0.645 & 3.612 & 0.456 & 2.284 & 0.881 & 3.146 \\
\hline${ }^{2} \mathbf{F}_{\mathbf{1}}$ & -0.448 & 2.706 & 0.473 & 2.456 & 1.176 & 4.751 & 1.117 & 4.690 \\
\hline${ }^{\mathbf{2}} \mathbf{F}_{\mathbf{1}} \mathbf{M}_{\mathbf{1}}$ & -0.238 & 2.737 & 0.422 & 2.981 & 0.437 & 3.268 & 0.878 & 3.397 \\
\hline${ }^{\mathbf{3}} \mathbf{F}_{\mathbf{1}}$ & 0.289 & 2.768 & 0.325 & 2.287 & 0.582 & 2.968 & 0.934 & 3.346 \\
\hline${ }^{3} \mathbf{F}_{\mathbf{1}} \mathbf{M}_{\mathbf{1}} \mathbf{M}_{\mathbf{1}}$ & -0.180 & 2.135 & 0.280 & 1.990 & 0.532 & 2.628 & 1.276 & 4.828 \\
\hline
\end{tabular}


Fig.1\&2 Frequency curves of plant height in F1M1 of Nirmala X Prachi and Frequency curves of plant height in F1M1 of Nirmala X Amrit
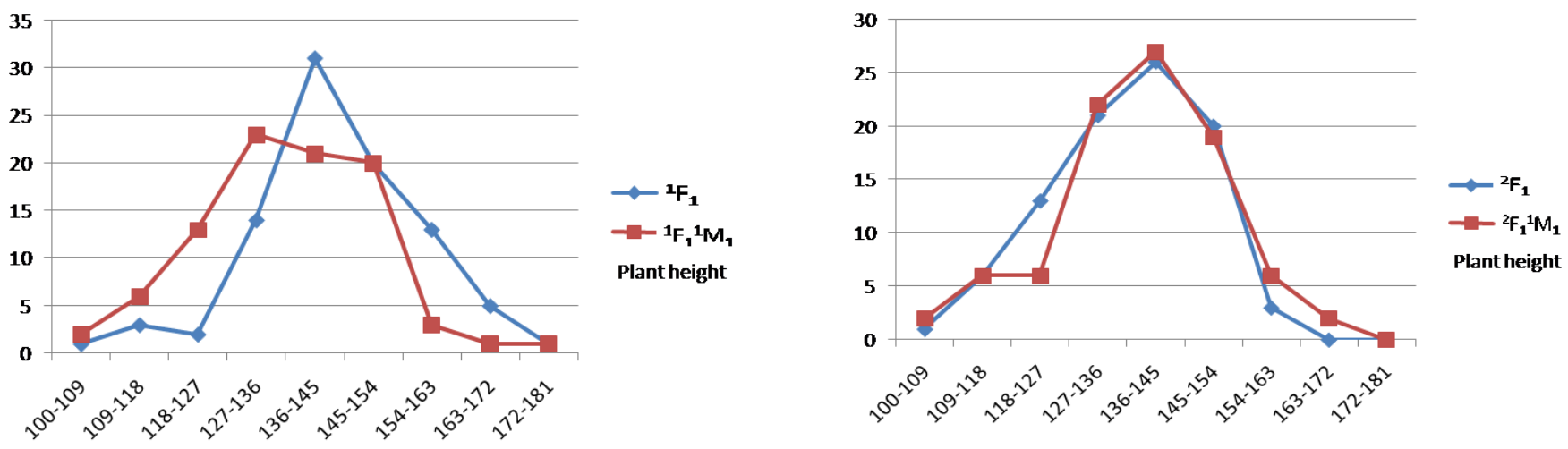

Fig.3\&4 Frequency curves of plant height in F1M1 of Prachi X Amrit \& Frequency curves of primary branches/plant in F1M1 of Nirmala X Prachi
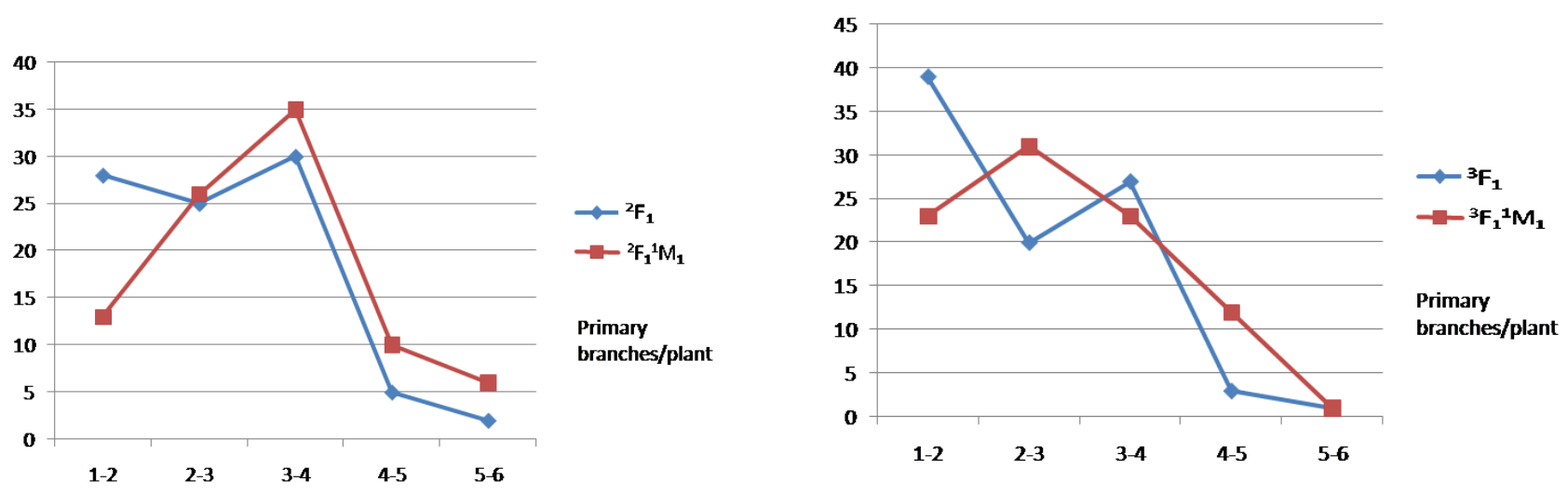

Fig.5\&6 Frequency curves of primary branches/plant in F1M1 of Nirmala X Amrit \& Frequency curves of primary branches/plant in F1M1 of Prachi X Amrit
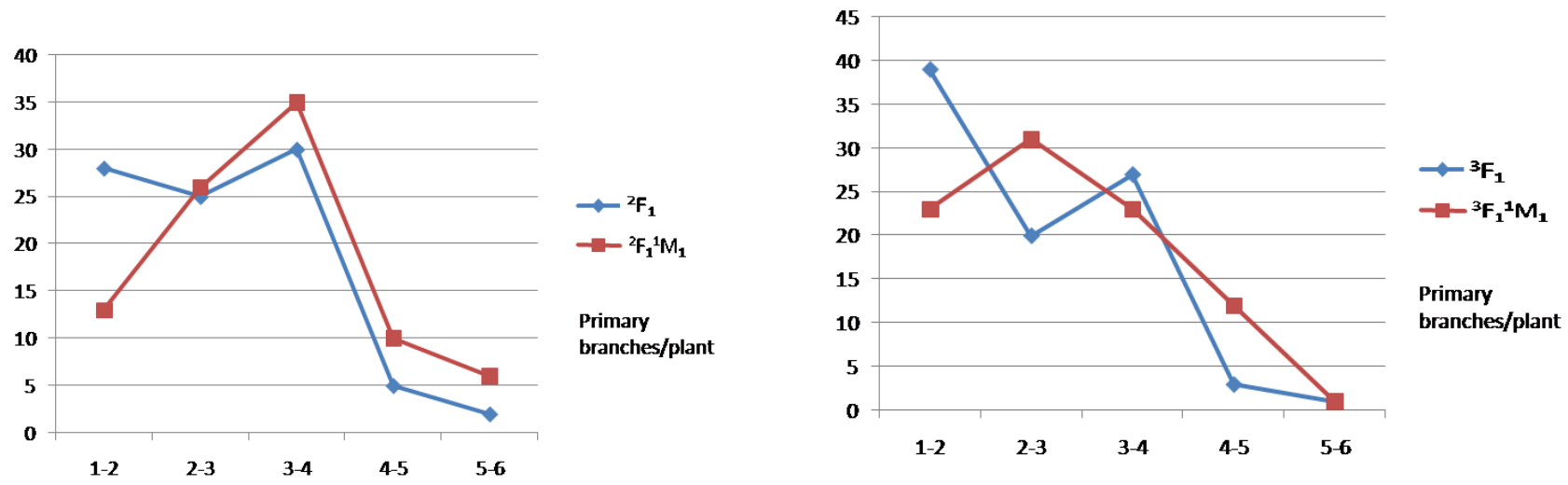
Fig.7\&8 Frequency curves of capsules/plant in F1M1 of Nirmala X Prachi \& Frequency curves of capsules/plant in F1M1 of Nirmala X Amrit
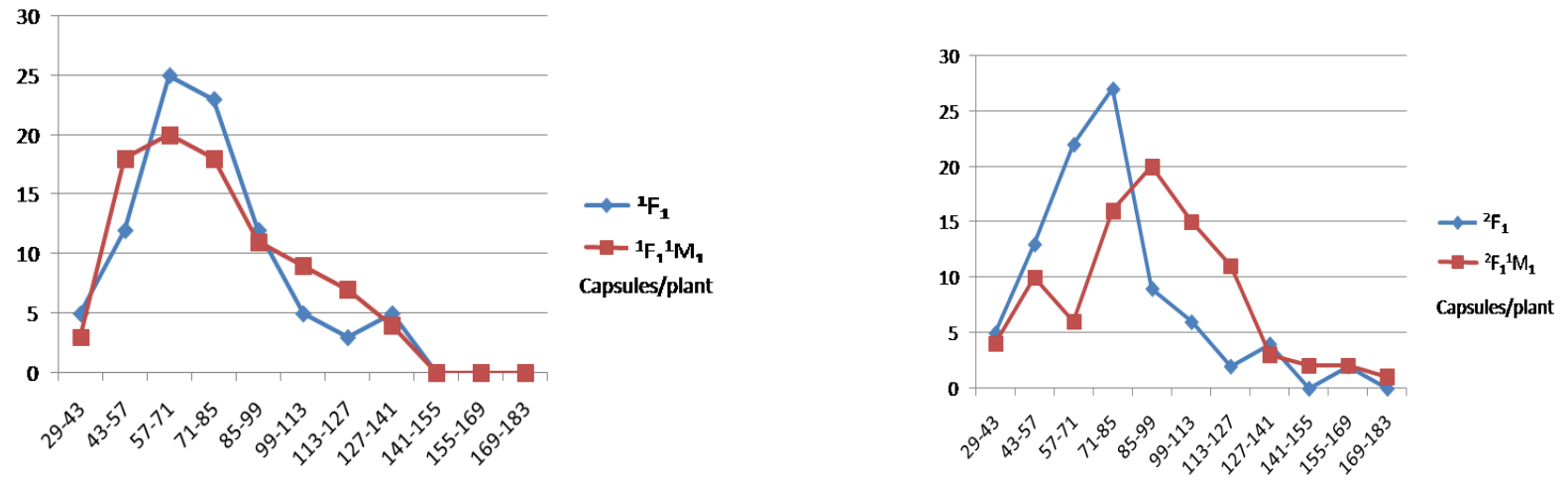

Fig.9\&10 Frequency curves of capsules/plant in F1M1 of Prachi X Amrit \& Frequency curves of seed yield/plant in F1M1 of Nirmala X Prachi
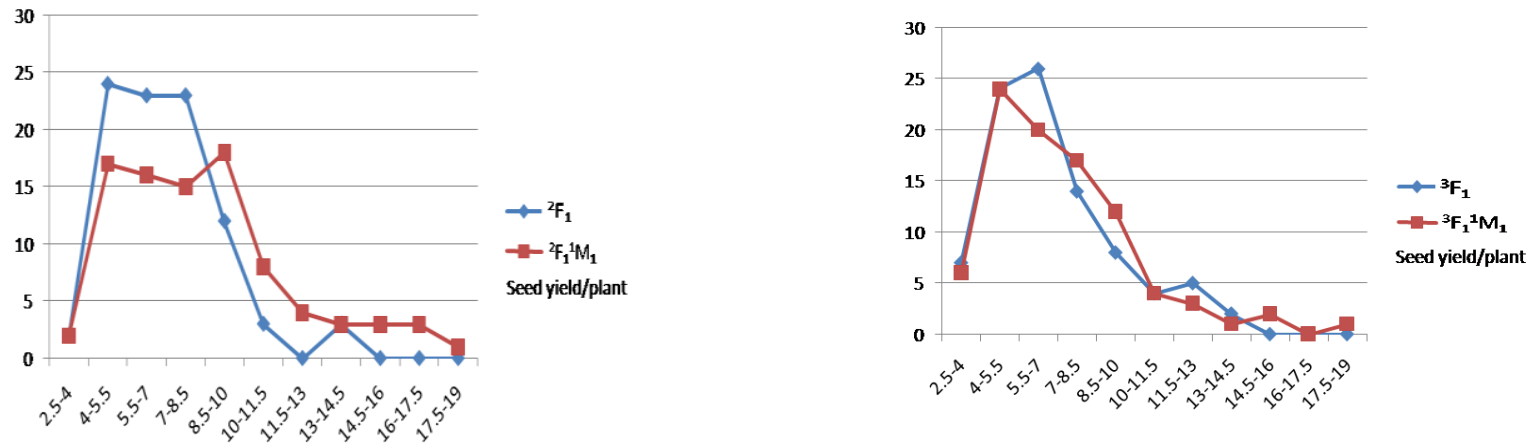

Fig.11\&12 Frequency curves of seed yield/plant in F1M1 of Nirmala X Amrit \& Frequency curves of seed yield/plant in F1M1 of Prachi X Amrit

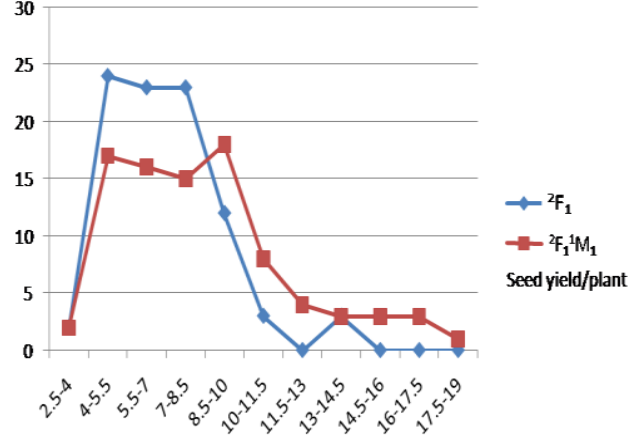

Frequency curves showed more or less restricted distribution in mutant population for primary branches per plant than their respective controls. Skewness and kurtosis

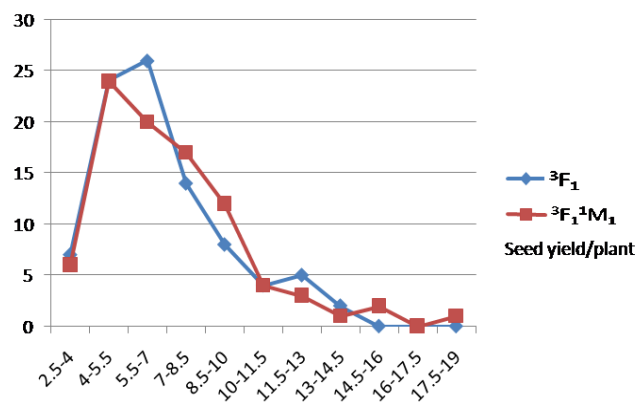

estimates of primary branches per plant appeared to be positive skewness, longer tail to the right in the $\mathrm{F}_{1}{ }^{1} \mathrm{M}_{1 \mathrm{~s}}$. One cycle EMS treated $F_{1}{ }^{1} M_{1}$ of Nirmala $X$ Prachi showed 
moderately skewness to the right and slightly leptokurtic distribution. Rest two $\mathrm{F}_{1}{ }^{1} \mathrm{M}_{1 \mathrm{~s}}$ gave slight positive skewness, longer tail to the right and platykurtic distribution of primary branches per plant. $\mathrm{F}_{1}{ }^{1} \mathrm{M}_{1}$ of Prachi X Amrit Showed kurtosis estimates of 1.990, indicating that the distribution is more platykurtic, i.e., lesser peaked than normal.

In frequency curves mutant population showed slight greater spread than their respective controls for number of capsules per plant. All the three $\mathrm{F}_{1}{ }^{1} \mathrm{M}_{1 \mathrm{~s}}$ gave a slight positive skewness, longer tail to the right in the distribution for number of capsules per plant. $\mathrm{F}_{1}{ }^{1} \mathrm{M}_{1}$ of Nirmala $\mathrm{X}$ Prachi showed kurtosis estimates of 2.284, indicating slight platykurtic nature of the population.

The distribution of seed yield per plant appeared to be positive skewness, longer tail to the right in all the $\mathrm{F}_{1}{ }^{1} \mathrm{M}_{1 \mathrm{~s}}$ this is also apparent from the frequency curves. Population $\mathrm{F}_{1}{ }^{1} \mathrm{M}_{1}$ (mutant population of Prachi X Amrit) appeared to be highly skewed and leptokurtic in nature. Rest two $\mathrm{F}_{1}{ }^{1} \mathrm{M}_{1 \mathrm{~s}}$ showed moderately skewed and nearly mesokurtic distribution of seed yield per plant. Brock (1967) reported mutagenic treatments generally increased both skewness and kurtosis. Goud (1967) studied the differential response of gamma treatment in wheat for skewness and kurtosis. These changes in mutant population for different characters might be due to discrepancy expression of a trait induced by mutation. However, these changes would be better known from analyses in later generations.

\section{References}

Anonymous. 2015. Area, Production and Productivity. www.mahaagri.org.in

Brock, R. D. 1967. Quantitative variation in Arabidopsis thaliana induced by ionizing radiation. Radiat. Bot. 10: 209233.

FAOSTAT.2017. http://www.fao.org/faostat/ en

Goud, J.V.1967. Effect of chronic irradiation on bread wheat. Indian Journalof Genetics and Plant breeding. 27: 305309.

Gupta, S.C.1981. Fundamentals of Statistics. Himalaya Publishing House, Bouding. P.418-464.

Kumari, V., Chaudhary, H.K., Prasad, R., Kumar, A., Singh, A., Jambhulkar, S., Sanju,S.2016. Frequency and Spectrum of Mutations induced by Gamma radiations and Ethyl methane sulphonate in Sesame (Sesamum indicum L.). Scientia Agriculturae. 14 (3): 270-278.

Tamina Begum and Tapash Dasgupta. 2010.A comparison of the effects of physical and chemical mutagens in sesame (Sesamum indicum L.). Genetics and Molecular Biology. 33 (4): 761-766.

Tamina Begum and Tapash Dasgupta.2015. Amelioration of seed yield, oil content and oil quality through induced mutagenesis in sesame (Sesamum indicum). Bangladesh Journal of Botany. 44(1): 15-22.

\section{How to cite this article:}

Rajesh Kumar Kar, Tapash Kumar Mishra and Banshidhar Pradhan. 2019. Studies on Frequency Distribution, Skewness and Kurtosis in F1m1 Mutant Populations of Sesame. Int.J.Curr.Microbiol.App.Sci. 8(04): 1755-1760. doi: https://doi.org/10.20546/ijcmas.2019.804.204 